\title{
Measurement of the group velocity of light in sea water at the ANTARES site
}

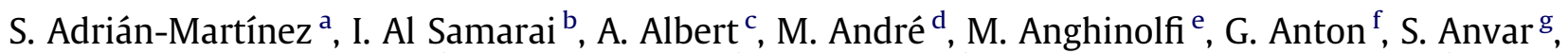
M. Ardid ${ }^{\mathrm{a}}$, A.C. Assis Jesus ${ }^{\mathrm{h}}$, T. Astraatmadja ${ }^{\mathrm{h}, 1}$, J-J. Aubert ${ }^{\mathrm{b}}$, B. Baret ${ }^{\mathrm{i}}, \mathrm{S}$. Basa ${ }^{\mathrm{j}}$, V. Bertin ${ }^{\mathrm{b}}$, S. Biagi ${ }^{\mathrm{k}, \mathrm{l}}$, A. Bigi ${ }^{\mathrm{m}}$, C. Bigongiari ${ }^{\mathrm{n}}$, C. Bogazzi ${ }^{\mathrm{h}}$, M. Bou-Cabo ${ }^{\mathrm{a}}$, B. Bouhou ${ }^{\mathrm{i}}$, M.C. Bouwhuis ${ }^{\mathrm{h}}$, J. Brunner ${ }^{\mathrm{b}, 2}$, J. Busto $^{\mathrm{b}}$,

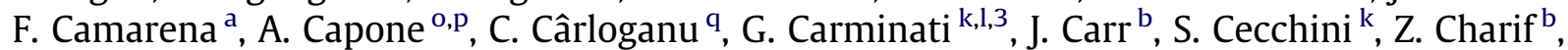

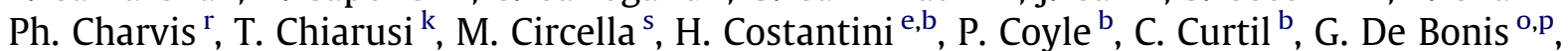
M.P. Decowski ${ }^{\text {h }}$, I. Dekeyser ${ }^{\mathrm{t}}$, A. Deschamps ${ }^{\mathrm{r}}$, C. Distefano ${ }^{\mathrm{u}}$, C. Donzaud ${ }^{\mathrm{i}, \mathrm{v}}$, D. Dornic ${ }^{\mathrm{n}}$, Q. Dorosti ${ }^{\mathrm{w}}$, D. Drouhin ${ }^{\mathrm{c}}$, T. Eberl ${ }^{\mathrm{f}}$, U. Emanuele ${ }^{\mathrm{n}}$, A. Enzenhöfer ${ }^{\mathrm{f}}$, J-P. Ernenwein ${ }^{\mathrm{b}}$, S. Escoffier ${ }^{\mathrm{b}}$, P. Fermani ${ }^{\mathrm{o}, \mathrm{p}}$, M. Ferri ${ }^{\mathrm{a}}$, V. Flaminio ${ }^{\mathrm{m}, \mathrm{x}}$, F. Folger ${ }^{\mathrm{f}}$, U. Fritsch ${ }^{\mathrm{f}}$, J-L. Fuda ${ }^{\mathrm{t}}$, S. Galatà ${ }^{\mathrm{b}}$, P. Gay ${ }^{\mathrm{q}}$, K. Geyer ${ }^{\mathrm{f}}$, G. Giacomelli ${ }^{\mathrm{k}, \mathrm{l}}$, V. Giordano ", J.P. Gómez-González ${ }^{\mathrm{n}}$, K. Graf ${ }^{\mathrm{f}}$, G. Guillard ${ }^{\mathrm{q}}$, G. Halladjian ${ }^{\text {b }}$, G. Hallewell ${ }^{\mathrm{b}}$, H. van Haren ${ }^{\mathrm{y}}$, J. Hartman ${ }^{\text {h}}$, A.J. Heijboer ${ }^{\text {h}}$, Y. Hello ${ }^{\mathrm{r}}$, J.J. Hernández-Rey ${ }^{\mathrm{n}}$, B. Herold ${ }^{\mathrm{f}}$, J. Hößl ${ }^{\mathrm{f}}$, C.C. Hsu ${ }^{\text {h}}$, M. de Jong ${ }^{\text {h,1 }}$, M. Kadler ${ }^{\text {z }}$, O. Kalekin ${ }^{\text {f }}$, A. Kappes ${ }^{f}$, U. Katz ${ }^{\text {f }}$, O. Kavatsyuk ${ }^{\text {w }}$, P. Kooijman ${ }^{\text {h,aa,ab }}$, C. Kopper ${ }^{\text {h,f }}$, A. Kouchner ${ }^{\mathrm{i}}$, I. Kreykenbohm ${ }^{\text {z }}$, V. Kulikovskiy ac,e, R. Lahmann ${ }^{\text {f }}$, P. Lamare ${ }^{\mathrm{g}}$, G. Larosa ${ }^{\mathrm{a}}$, D. Lattuada ${ }^{\mathrm{u}}$, D. Lefèvre ${ }^{t}, G$. Lim $^{\text {h,ab }}$, D. Lo Presti ${ }^{\text {ad,ae }}$, H. Loehner ${ }^{w}$, S. Loucatos ${ }^{\text {af }}$, S. Mangano ${ }^{n, *}$, M. Marcelin ${ }^{j}$, A. Margiotta ${ }^{\mathrm{k}, \mathrm{l}}$, J.A. Martínez-Mora ${ }^{\mathrm{a}}$, J.E. McMillan ag, A. Meli ${ }^{\mathrm{f}}$, T. Montaruli ${ }^{\mathrm{s}, \mathrm{ah}}$, L. Moscoso ${ }^{\mathrm{i}, \mathrm{af}, 4}, \mathrm{H}^{\mathrm{g}}$ Motz $^{\mathrm{f}}$, M. Neff ${ }^{f}$, E. Nezri ${ }^{j}$, D. Palioselitis ${ }^{\text {h }}$, G.E. Păvălaş ${ }^{\text {ai }}$, K. Payet ${ }^{\text {af }}$, P. Payre ${ }^{\text {b, }}$, J. Petrovic ${ }^{\text {h }}$, P. Piattelli ${ }^{\text {u }}$,

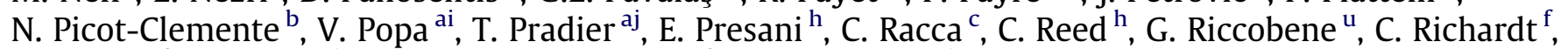

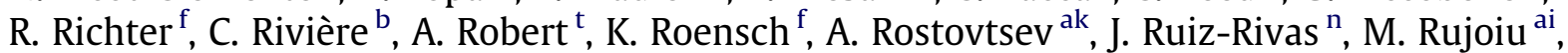
G.V. Russo ad,ae, F. Salesa ${ }^{\text {n}}$, D.F.E. Samtleben ${ }^{\text {h}}$, P. Sapienza ${ }^{\text {u}}$, F. Schöck ${ }^{\mathrm{f}}$, J-P. Schuller ${ }^{\text {af }}$, F. Schüssler ${ }^{\text {af }}$, T. Seitz ${ }^{\mathrm{f}}$, R. Shanidze ${ }^{\mathrm{f}}$, F. Simeone ${ }^{\text {o,p }}$, A. Spies ${ }^{\mathrm{f}}$, M. Spurio ${ }^{\text {k,1, J.J.M. Steijger }}{ }^{\text {h }}$, Th. Stolarczyk ${ }^{\text {af }}$, A. Sánchez-Losa ${ }^{\mathrm{n}}$, M. Taiuti ${ }^{\mathrm{e}, \mathrm{al}}$, C. Tamburini ${ }^{\mathrm{t}}$, L.F. Thompson ${ }^{\mathrm{ag}}, \mathrm{S}$. Toscano ${ }^{\mathrm{n}}$, B. Vallage ${ }^{\text {af }}$, V. Van Elewyck ${ }^{\mathrm{i}}$, G. Vannoni af, M. Vecchi ${ }^{\mathrm{b}}$, P. Vernin ${ }^{\text {af }}$, S. Wagner ${ }^{\mathrm{f}}$, G. Wijnker ${ }^{\mathrm{h}}$, J. Wilms ${ }^{\mathrm{z}}$, E. de Wolf ${ }^{\text {h,ab }}, H$. Yepes ${ }^{n}$, D. Zaborov ${ }^{\text {ak }}$, J.D. Zornoza ${ }^{n}$, J. Zúñiga ${ }^{n}$

\footnotetext{
a Institut d'Investigació per a la Gestió Integrada de les Zones Costaneres (IGIC) - Universitat Politècnica de València. C/ Paranimf 1, 46730 Gandia, Spain ${ }^{\mathrm{b}}$ CPPM, Aix-Marseille Université, CNRS/IN2P3, Marseille, France

${ }^{\mathrm{c}}$ GRPHE - Institut universitaire de technologie de Colmar, 34 rue du Grillenbreit, BP 50568, 68008 Colmar, France

${ }^{\mathrm{d}}$ Technical University of Catalonia, Laboratory of Applied Bioacoustics, Rambla Exposició, 08800 Vilanova i la Geltrú, Barcelona, Spain

e INFN - Sezione di Genova, Via Dodecaneso 33, 16146 Genova, Italy

${ }^{\mathrm{f}}$ Friedrich-Alexander-Universität Erlangen-Nürnberg, Erlangen Centre for Astroparticle Physics, Erwin-Rommel-Str. 1, 91058 Erlangen, Germany

${ }^{\mathrm{g}}$ Direction des Sciences de la Matière - Institut de recherche sur les lois fondamentales de l'Univers - Service d'Electronique des Détecteurs et d'Informatique, CEA Saclay,

91191 Gif-sur-Yvette Cedex, France

${ }^{\mathrm{h}}$ Nikhef, Science Park, Amsterdam, The Netherlands

${ }^{\mathrm{i}}$ APC - Laboratoire AstroParticule et Cosmologie, UMR 7164 (CNRS, Université Paris 7 Diderot, CEA, Observatoire de Paris) 10, rue Alice Domon et Léonie Duquet,

75205 Paris Cedex 13, France

${ }^{\mathrm{j}}$ LAM - Laboratoire d'Astrophysique de Marseille, Pôle de l'Étoile Site de Château-Gombert, rue Frédéric Joliot-Curie 38, 13388 Marseille Cedex 13, France

${ }^{\mathrm{k}}$ INFN - Sezione di Bologna, Viale Berti-Pichat 6/2, 40127 Bologna, Italy

${ }^{1}$ Dipartimento di Fisica dell'Università, Viale Berti Pichat 6/2, 40127 Bologna, Italy

${ }^{\mathrm{m}}$ INFN - Sezione di Pisa, Largo B. Pontecorvo 3, 56127 Pisa, Italy

${ }^{\mathrm{n}}$ IFIC - Instituto de Física Corpuscular, Edificios Investigación de Paterna, CSIC - Universitat de València, Apdo. de Correos 22085, 46071 Valencia, Spain

${ }^{\circ}$ INFN - Sezione di Roma, P.le Aldo Moro 2, 00185 Roma, Italy

P Dipartimento di Fisica dell'Università La Sapienza, P.le Aldo Moro 2, 00185 Roma, Italy

${ }^{\mathrm{q}}$ Clermont Université, Université Blaise Pascal, CNRS/IN2P3, Laboratoire de Physique Corpusculaire, BP 10448, 63000 Clermont-Ferrand, France

${ }^{r}$ Géoazur - Université de Nice Sophia-Antipolis, CNRS/INSU, IRD, Observatoire de la Côte d'Azur and Université Pierre et Marie Curie, BP 48,06235 Villefranche-sur-mer, France ${ }^{\mathrm{s}}$ INFN - Sezione di Bari, Via E. Orabona 4, 70126 Bari, Italy

${ }^{\mathrm{t}}$ COM - Centre d'Océanologie de Marseille, CNRS/INSU et Université de la Méditerranée, 163 Avenue de Luminy, Case 901, 13288 Marseille Cedex 9, France

" INFN - Laboratori Nazionali del Sud (LNS), Via S. Sofia 62, 95123 Catania, Italy

${ }^{\mathrm{v}}$ Univ Paris-Sud, 91405 Orsay Cedex, France

${ }^{\mathrm{w}}$ Kernfysisch Versneller Instituut (KVI), University of Groningen, Zernikelaan 25, 9747 AA Groningen, The Netherlands

${ }^{x}$ Dipartimento di Fisica dell'Università, Largo B. Pontecorvo 3, 56127 Pisa, Italy

${ }^{\mathrm{y}}$ Royal Netherlands Institute for Sea Research (NIOZ), Landsdiep 4, 1797 SZ 't Horntje (Texel), The Netherlands
} 
${ }^{\mathrm{z}}$ Dr. Remeis-Sternwarte and ECAP, Universität Erlangen-Nürnberg, Sternwartstr. 7, 96049 Bamberg, Germany

aa Universiteit Utrecht, Faculteit Betawetenschappen, Princetonplein 5, 3584 CC Utrecht, The Netherlands

${ }^{\mathrm{ab}}$ Universiteit van Amsterdam, Instituut voor Hoge-Energie Fysika, Science Park 105, 1098 XG Amsterdam, The Netherlands

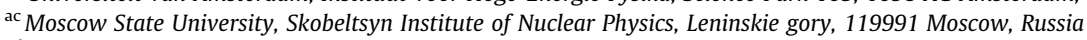

ad INFN - Sezione di Catania, Viale Andrea Doria 6, 95125 Catania, Italy

ae Dipartimento di Fisica ed Astronomia dell'Università, Viale Andrea Doria 6, 95125 Catania, Italy

af Direction des Sciences de la Matière - Institut de recherche sur les lois fondamentales de l'Univers - Service de Physique des Particules, CEA Saclay, 91191 Gif-sur-Yvette Cedex, France

ag Department of Physics and Astronomy, University of Sheffield, Hicks Building, Hounsfield Road, Sheffield S3 7RH, UK

ah DPNC, Université de Genève, Quai Ernest-Ansermet 24, 1211 Genève, Switzerland

ai Institute for Space Sciences, R-77125 Bucharest, Măgurele, Romania

aj IPHC-Institut Pluridisciplinaire Hubert Curien - Université de Strasbourg et CNRS/IN2P3 23 rue du Loess, BP 28, 67037 Strasbourg Cedex 2, France

ak ITEP - Institute for Theoretical and Experimental Physics, B. Cheremushkinskaya 25, 117218 Moscow, Russia

al Dipartimento di Fisica dell'Università, Via Dodecaneso 33, 16146 Genova, Italy

\section{A R T I C L E I N F O}

Article history:

Received 27 October 2011

Received in revised form 19 December 2011

Accepted 31 December 2011

Available online 12 January 2012

Keywords:

ANTARES

Neutrino telescope

Optical beacon system

Velocity of light

Refractive index

\section{A B S T R A C T}

The group velocity of light has been measured at eight different wavelengths between $385 \mathrm{~nm}$ and $532 \mathrm{~nm}$ in the Mediterranean Sea at a depth of about $2.2 \mathrm{~km}$ with the ANTARES optical beacon systems. A parametrisation of the dependence of the refractive index on wavelength based on the salinity, pressure and temperature of the sea water at the ANTARES site is in good agreement with these measurements.

(c) 2012 Elsevier B.V. All rights reserved.

\section{Introduction}

The ANTARES neutrino telescope is located on the bottom of the Mediterranean Sea $\left(42^{\circ} 50^{\prime} \mathrm{N}, 6^{\circ} 10^{\prime} \mathrm{E}\right)$ at a depth of $2475 \mathrm{~m}$, roughly $40 \mathrm{~km}$ offshore from Toulon in France. The main objective of the experiment is the observation of neutrinos of cosmic origin in the southern hemisphere sky. Sea water is used as the detection medium of the Cherenkov light induced by relativistic charged particles resulting from the interaction of neutrinos. The particle trajectory is reconstructed from the measured arrival times of the detected photons. The detector consists of 885 photomultiplier tubes (PMTs) mounted on twelve vertical lines with a length of about $450 \mathrm{~m}$. The horizontal separation between lines is about $70 \mathrm{~m}$. Further details can be found elsewhere [1-3].

Charged particles traveling through sea water produce the emission of Cherenkov light whenever the velocity of the particle exceeds that of light in water. The Cherenkov photons are emitted at a characteristic angle, $\theta_{c}$, with respect to the particle direction. This angle is related to the index of refraction of the medium as $\cos \theta_{c}=\frac{1}{\beta n_{p}}$. In this, $\beta$ is the velocity of the particle relative to the speed of light in vacuum. The index of refraction, $n_{p}$, corresponds to the ratio between the speed of light in vacuum and the phase velocity of light in water. The individual photons then travel through the water at the group velocity. Both the phase and the group velocity depend on the wavelength of the photons. This is usually referred to as chromatic dispersion. The group velocity is related to its phase velocity in the following way:

$n_{g}=\frac{n_{p}}{1+\frac{\lambda}{n_{p}} \frac{d n_{p}}{d \lambda}}$

\footnotetext{
* Corresponding author.

E-mail address: manganos@ific.uv.es (S. Mangano).

1 Also at University of Leiden, The Netherlands.

2 On leave at DESY, Platanenallee 6, D-15738 Zeuthen, Germany.

3 Now at University of California, Irvine, 92697 CA, USA.

4 Deceased.
}

where $\lambda$ is the wavelength of light. The index of refraction, $n_{g}$, corresponds to the ratio between the speed of light in vacuum and the group velocity of light in water.

Since the PMTs cannot distinguish the photon wavelength, the variation of the photon emission angle and the group velocity due to chromatic dispersion cannot be accounted for on the individual photon level. Nevertheless, the average effect of the wavelength dependencies are accounted for in the algorithm used to reconstruct the particle trajectory $[4,5]$.

A measurement of the group velocity of light has been made using the optical beacon system of ANTARES. This system consists of a set of pulsed light sources (LEDs and lasers) which are distributed throughout the detector and illuminate the PMTs with short duration flashes of light. The refractive index is deduced from the recorded time of flight distributions of photons at different distances from the sources for eight different wavelengths between $385 \mathrm{~nm}$ and $532 \mathrm{~nm}$.

\section{Experimental setup}

The PMTs of ANTARES are sensitive to photons in the wavelength range between $300 \mathrm{~nm}$ and $600 \mathrm{~nm}$. The maximum quantum efficiency is about $22 \%$ between $350 \mathrm{~nm}$ and $450 \mathrm{~nm}$. The arrival time and integrated charge of the analogue pulse from the PMT are measured by the readout electronics [6]. The transit time spread of single photo-electrons of the PMT is around $3.5 \mathrm{~ns}$ (FWHM) [7].

The group velocity of light has been measured using the ANTARES optical beacon system. This system was primarily designed to perform time calibration in situ $[7,8]$. There are two types of optical beacons, the LED optical beacons and the laser beacons. There are four LED optical beacons distributed along each detector line and two laser beacons at the bottom of two central lines. The in situ measurement of the temperature and salinity is provided by some conductivity/temperature/depth sensors. ${ }^{5}$

\footnotetext{
5 SEABIRD CTD (SBE37-SMP), http://www.seabird.com/.
} 

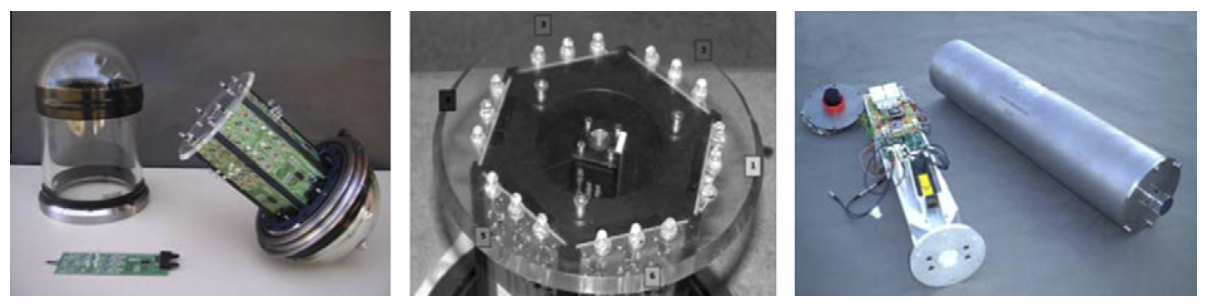

Fig. 1. Picture of a standard LED optical beacon (left), the modified LED optical beacon (middle) and a laser beacon (right).

A standard LED optical beacon contains 36 individual LEDs distributed over six vertical faces forming a hexagonal cylinder housed in a pressure resistant glass enclosure (Fig. 1, left). On each face, five LEDs point radially outwards and one upwards. All LEDs emit light at an average wavelength of $469 \mathrm{~nm}$, except the two LEDs located on the lowest LED optical beacon of Line 12 which emit light at an average wavelength of $400 \mathrm{~nm}$. A modified LED optical beacon was installed in 2010 on Line 6. This LED optical beacon has three LEDs per face instead of six, all of them pointing upwards (Fig. 1, centre). The three LEDs of each face emit light of the same colour. The average wavelength of the light from the six faces are $385,400,447,458,494$ and $518 \mathrm{~nm}$. The LEDs emit light with a maximum intensity of about $160 \mathrm{pJ}$ and a pulse width of about $4 \mathrm{~ns}$ FWHM. The intensity of the emitted light can be varied changing the voltage feeding the LEDs.

The laser beacon consists of a Nd-YAG solid state laser (Fig. 1, right). It can emit pulses of light with an intensity of about $1 \mu \mathrm{J}$ and pulse width of about 0.8 ns FWHM. The average wavelength is $532 \mathrm{~nm}$. This light is spread out by an optical diffuser, so that the light can reach the surrounding lines. During calibration runs, the LEDs and lasers flash at a frequency of $330 \mathrm{~Hz}$. Further details about the optical beacon system can be found elsewhere [7-9].

The wavelength spectra of the light sources used for this analysis were measured using a calibrated spectrometer $^{6}$ (see Fig. 2a). The typical width of each spectrum is around $10 \mathrm{~nm}$ except for the green LED (518 nm), which is larger, and the laser (532 nm), which is much smaller.

Due to the wavelength dependence of the absorption of light in water, the spectra change as a function of the distance travelled by the light. The expected wavelength distributions as a function of distance have been estimated by Monte Carlo simulations using the dependence of absorption length on wavelength given by Smith and Baker [10]. In Fig. 2b the spectra at a distance of $120 \mathrm{~m}$ are shown for the different light sources. In particular, absorption has a large effect for wavelengths above $500 \mathrm{~nm}$. Notice that the distributions have been renormalised to unity in each peak and therefore the relative effect of absorption between sources is not observed. This renormalisation is performed in order to show the change in the shapes of the distributions, which is what influences the velocity measurement. The evolution of the spectra is taken into account in the final results (Section 4), in particular the uncertainty assigned to the wavelengths has been taken to be the root mean square (RMS) of the wavelength distribution given by the simulation.

\section{Data acquisition and analysis}

In order to measure the optical properties of the deep sea water, designated data taking runs were performed using the optical beacon system. During these runs, one single LED located in the lowest optical beacon of a line and pointing upward was flashed. Only the signals recorded by the PMTs along the same line are used in the

\footnotetext{
${ }^{6}$ Ocean Optics HR4000CG-UV-NIR, http://www.oceanoptics.com/.
}

analysis. As a result, the line movements due to the sea currents can safely be ignored.

The runs used in this analysis were taken between May 2008 and April 2011. Each run contains typically more than 100,000 light flashes. Each flash is detected by a small PMT inside the optical beacon. The time of the flash and the arrival times of the photons on the PMTs were recorded within a time window from $1500 \mathrm{~ns}$ before to $1500 \mathrm{~ns}$ after the flash. The integrated charge of the analogue pulses of the PMTs were also recorded. Only runs were used when the average rate of background light was below $100 \mathrm{kHz}$.

In Fig. 3, the distribution of the arrival times of photons on a PMT located $100 \mathrm{~m}$ above the LED optical beacon $(\lambda=469 \mathrm{~nm})$ is shown. The time, $t=0 \mathrm{~ns}$, corresponds to the time of the flash. A clear peak at $t=470 \mathrm{~ns}$ can be seen which corresponds to the shortest propagation time of the light. The tail with late photons can be attributed to light scattering. The flat background arises from the optical background due to ${ }^{40} \mathrm{~K}$ decays and bioluminescence.

A convolution of a Gaussian and an exponential distribution on top of a flat background is fitted to the data. The Gaussian distribution reproduces the transit time spread of the PMTs, the duration of the light flash and the effect of the chromatic dispersion in water. The exponential distribution takes into account the effect of the scattering of photons in water. The fit function can be formulated as:

$f(t)=B+S \cdot e^{-\frac{t-\mu}{\tau}} \times \operatorname{erfc}\left(\frac{1}{\sqrt{2}}\left(\frac{\sigma}{\tau}-\frac{t-\mu}{\sigma}\right)\right)$

where $t$ is the arrival time of the photons. The fit parameters are the optical background, $B$, the signal strength, $S$, the mean, $\mu$, and width, $\sigma$, of the Gaussian distribution and the exponential decay constant, $\tau$. In Eq. 2, $\operatorname{erfc}(t)$ is the complementary error function distribution. An example of the fit is shown in the inset of Fig. 3. The fit is determined in the range from 200 ns before the most populated bin and 20 ns after. The arrival time of the light flash at each PMT is estimated by the fitted mean value of the Gaussian distribution.

An example of the measured arrival times as a function of the distance between the optical beacon and the PMT is shown in Fig. 4a. A linear function has been fitted to the data to extract the group velocity of the light. In the fitting procedure, the distance between the light source and the PMT has been restricted to the range between $50 \mathrm{~m}$ and $250 \mathrm{~m}$ in order to limit the effects of time slewing [7] and optical background on the measurement.

A Monte Carlo simulation of the response of the detector to LED flashes has been made. The analysis method was performed to validate the analysis procedure and to study the systematic effects due to the assumed light absorption and scattering parameters. Table 1 shows the different contributions to the systematic uncertainty in the measurement of the refractive index. These contributions have been determined as follows. For the wavelength values of $400 \mathrm{~nm}, 470 \mathrm{~nm}$ and $532 \mathrm{~nm}$, the difference of the refractive index obtained after the fitting procedure with respect to the input refractive index is shown in the first row of Table 1 . This variation, which has been computed for the default values of absorption and 

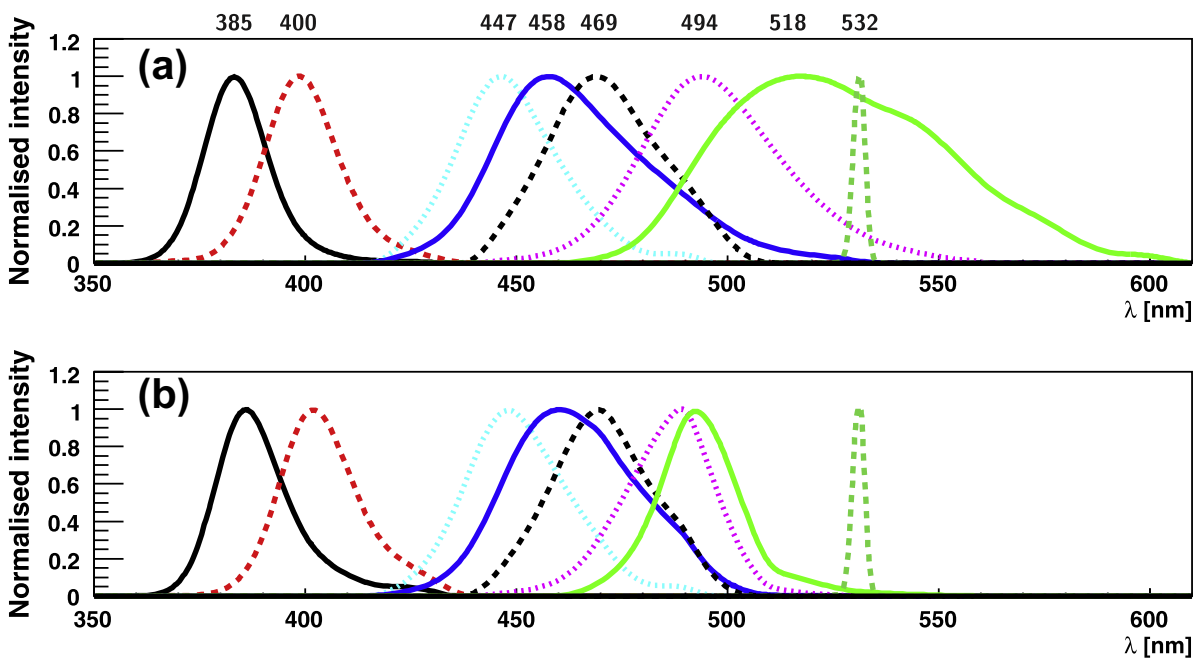

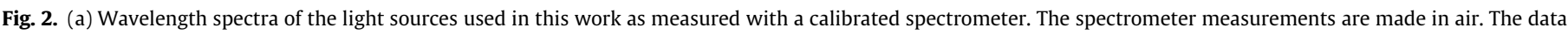

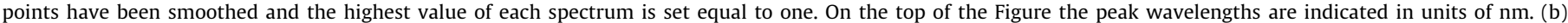

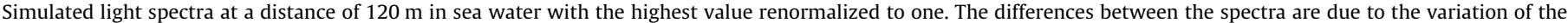
absorption length as a function of the wavelength.

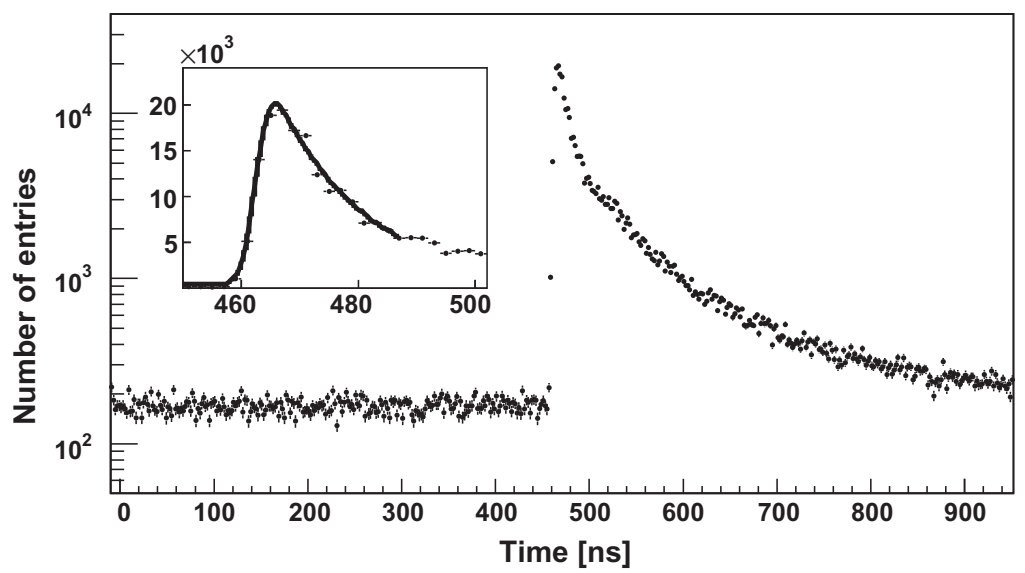

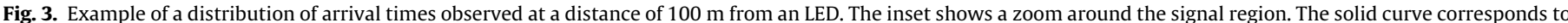
a fit function as described in the text and given in Eq. 2.
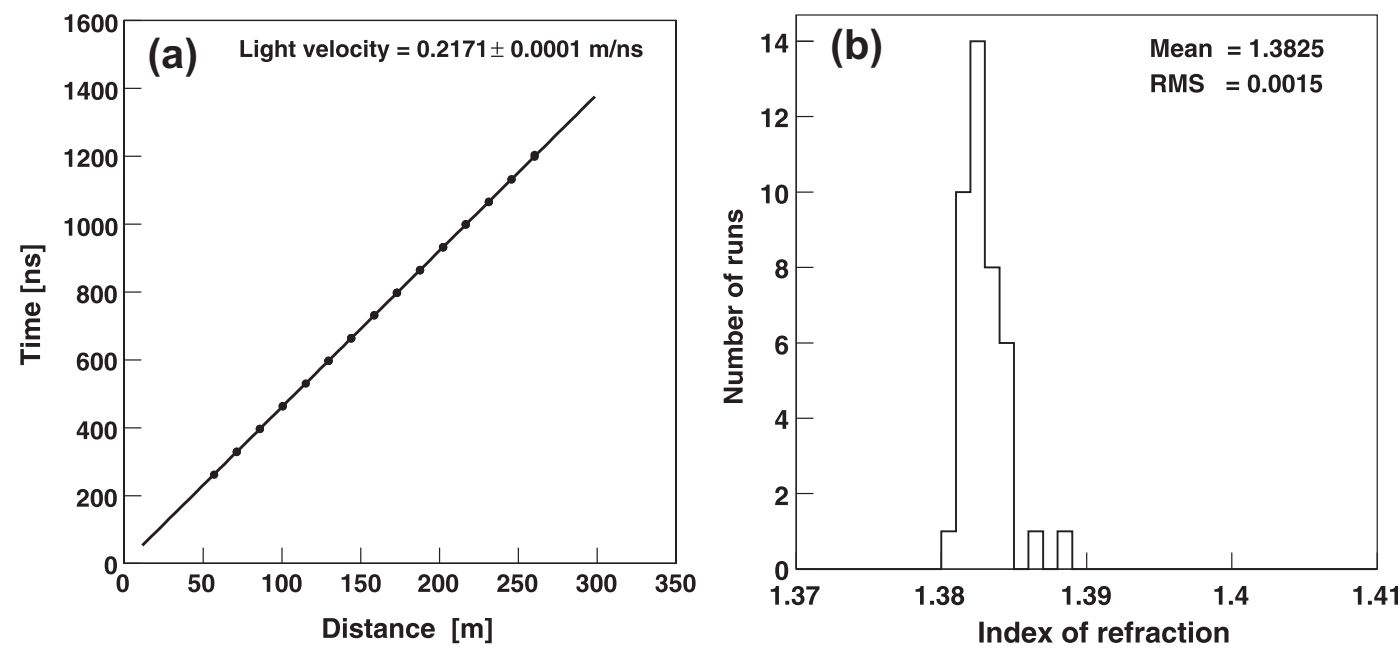

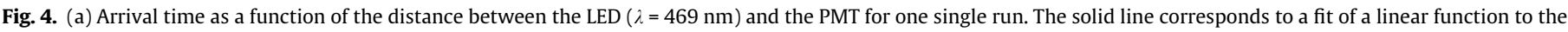
data (see text). (b) Distribution of the measured refractive index for a total of 42 runs. 
Table 1

The systematic uncertainty of the refractive index measurement is estimated for three different wavelengths by varying the absorption length and the scattering length (see text).

\begin{tabular}{|c|c|c|c|}
\hline Wavelength [nm] & 400 & 470 & 530 \\
\hline Source of uncertainty & \multicolumn{3}{|c|}{ Variation in [\%] } \\
\hline Method & 0.27 & 0.13 & 0.04 \\
\hline Absorption length & 0.11 & 0.05 & 0.07 \\
\hline Scattering length & 0.22 & 0.34 & 0.24 \\
\hline Total systematic uncertainty [\%] & \pm 0.37 & \pm 0.37 & \pm 0.25 \\
\hline
\end{tabular}

scattering lengths, is termed "Method". In the second row, the variation in percentage of the fitted value is shown when the absorption length changes by $\pm 50 \%$. Finally, in the third row the variation in percentage of the fitted value is shown when the scattering length is varied between $20 \mathrm{~m}$ and $70 \mathrm{~m}$ [11]. As can be seen, the latter is the largest contribution to the systematic uncertainty. Adding in quadrature these values one obtains a systematic error that varies from $0.25 \%$ to $0.37 \%$ depending on the wavelength.

\section{Determination of the refractive index}

Between May 2008 and March 2010, a total of 42 runs were taken using an LED with an average wavelength of $469 \mathrm{~nm}$. Three different LED intensities were used. For a high, middle or low intensity run the range of distances between the optical beacon and the PMT used in the fit were $50-250 \mathrm{~m}, 40-220 \mathrm{~m}$ and $10-130 \mathrm{~m}$, respectively. The measured refractive index values of these runs are shown in Fig. 4b. In addition to these runs, 14 runs using an LED with an average wavelength of $400 \mathrm{~nm}$ and 13 runs using an LED with an average wavelength of $532 \mathrm{~nm}$ were taken. Between November 2010 and April 2011 eight runs with a modified optical beacon were taken, extending the measurements with six additional wavelengths. The index of refraction is estimated at each wavelength by the mean of the distribution. The measured refractive indices with the systematic uncertainty are shown in Fig. 5 and tabulated in Table 2. As mentioned in Section 2, the uncertainties in the wavelengths have been taken to be the RMS of the corresponding distribution at the middle of the distance ranges. The variation of the RMS values in this range with respect to the middle is $\pm 2 \mathrm{~nm}$.

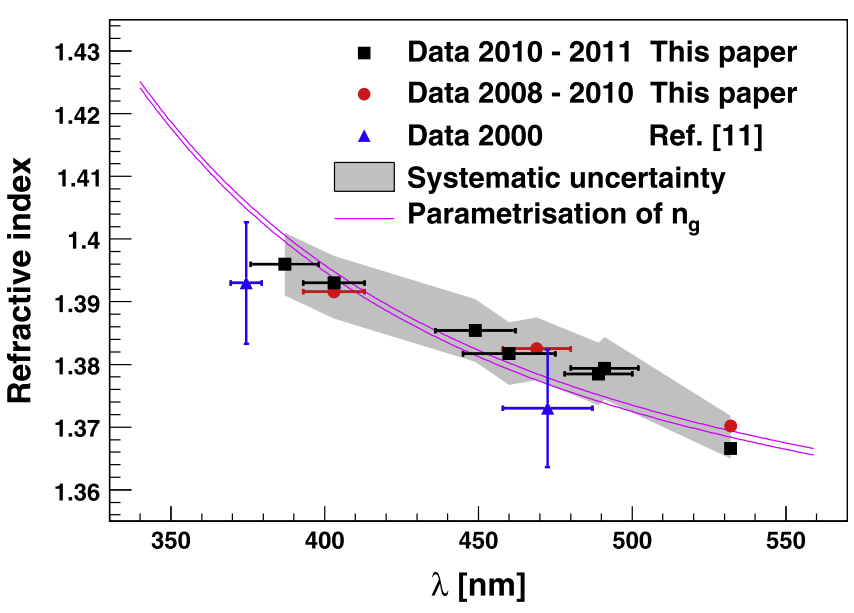

Fig. 5. Index of refraction corresponding to the group velocity of light as a function of the wavelength. Also shown are results from measurements made in [11]. The grey band shows the systematic uncertainty. The two solid lines correspond to a parametrisation of the index of refraction evaluated at a pressure of $200 \mathrm{~atm}$ (lower line) and $240 \mathrm{~atm}$ (upper line) (see text).
Table 2

Summary of the refractive index results for the 2008-2011 data shown in Fig. 5. For the refractive index first the statistical uncertainties are shown. They are computed as the RMS of the measured refractive index for each wavelength divided by the square root of the number of runs. In addition, there is a total systematic uncertainty of \pm 0.005 .

\begin{tabular}{clll}
\hline Wavelength $(\mathrm{nm})$ & Refractive index & Number of runs & Time period \\
\hline $403 \pm 10$ & $1.3916 \pm 0.0007$ & 14 & $2008-2010$ \\
$469 \pm 12$ & $1.3825 \pm 0.0002$ & 42 & $2008-2010$ \\
$532 \pm 1$ & $1.3702 \pm 0.0007$ & 13 & $2008-2010$ \\
$387 \pm 11$ & $1.3960 \pm 0.0007$ & 8 & $2010-2011$ \\
$403 \pm 10$ & $1.3930 \pm 0.0007$ & 8 & $2010-2011$ \\
$449 \pm 13$ & $1.3854 \pm 0.0003$ & 8 & $2010-2011$ \\
$460 \pm 15$ & $1.3817 \pm 0.0003$ & 8 & $2010-2011$ \\
$489 \pm 11$ & $1.3785 \pm 0.0003$ & 8 & $2010-2011$ \\
$491 \pm 11$ & $1.3794 \pm 0.0006$ & 8 & $2010-2011$ \\
$532 \pm 1$ & $1.3666 \pm 0.0006$ & 8 & \\
\hline
\end{tabular}

The velocity of light in sea water at a given wavelength depends on the temperature, the salinity and the pressure of the water, because the density of sea water depends on these variables. A parametrisation of the light velocity proposed by Quan and Fry [12] is based on data from Austin and Halikas [13]. This parametrisation was modified to incorporate a correction for pressure [11]. During the data taking period, the temperature and salinity were measured in situ at a depth of $2250 \mathrm{~m}$. At an ambient temperature of $T=12.9 \pm 0.1^{\circ} \mathrm{C}$ and salinity of $S=38.48 \pm 0.01 \%$, the refractive index corresponding to the phase velocity as a function of wavelength is expressed as:

$$
\begin{aligned}
n_{p}(\lambda, p)= & 1.32292+(1.32394-1.32292) \times \frac{p-200}{240-200} \\
& +\frac{16.2561}{\lambda}-\frac{4382}{\lambda^{2}}+\frac{1.1455 \times 10^{6}}{\lambda^{3}}
\end{aligned}
$$

where $\lambda$ is the wavelength (in units of $\mathrm{nm}$ ) and $p$ is the pressure (in units of atm). Using Eq. 1, the result of this parametrisation can be compared to the measurements (see Fig. 5).

From the known variations of temperature, salinity and pressure, the refractive index for a particular wavelength and at a given depth can be determined with an accuracy of better than $4 \times 10^{-5}$. The parametrisation is in good agreement with the measurements.

As mentioned in Section 1, the PMTs are unable to distinguish the wavelength of the incoming photons, so the effect of this chromatic dependence can only be taken into account on average. The spread of the arrival time residuals with respect to the expected arrival time of a $460 \mathrm{~nm}$ photon have been computed by means of a standalone Monte Carlo simulation using the phase velocity for the emission angle and the group velocity (as given by the Eqs. 3 and 1) for the arrival time. This simulation indicates that the spread of the time residual is $0.6 \mathrm{~ns}$ at $10 \mathrm{~m}, 1.6 \mathrm{~ns}$ at $40 \mathrm{~m}$, $2.7 \mathrm{~ns}$ at $100 \mathrm{~m}$ and $3.6 \mathrm{~ns}$ at $200 \mathrm{~m}$. The time uncertainty introduced by this spread is unavoidable and is taken into account in the ANTARES official simulation program [14,15]. Even though the exact influence of the medium depends on the particular Cherenkov photon (wavelength, distance to the hit PMT) and therefore requires a full simulation, a rough estimate of the average effect can be obtained assuming that a majority of hits are between $40 \mathrm{~m}$ and $100 \mathrm{~m}$ from the track, which gives a value of $\sim 2 \mathrm{~ns}$ for the uncertainty introduced by the transmission of light in sea water, including chromatic dispersion. This value is to be compared with $\sim 1.3$ ns coming from the PMTs transit time spread and to $\sim 1$ ns from time calibration. Monte Carlo simulation studies show also that an additional uncertainty of $\sim 2$ ns on the spread of the time residual degrades the neutrino pointing accuracy about 0.1 degrees. 


\section{Summary}

Using pulsed light sources with wavelengths between $385 \mathrm{~nm}$ and $532 \mathrm{~nm}$ the group velocity of light in sea water at the ANTARES site has been measured as a function of wavelength. The emission spectra determined in the laboratory for the different pulsed sources have been used as input to a Monte Carlo simulation in order to correct for the effect of absorption on the corresponding velocity measurement. Except for two sources these corrections are in general small. Likewise, a Monte Carlo simulation has been used to evaluate the systematic uncertainties and to check, that the procedure to obtain the speed of light is robust and unbiased. The results obtained for the dependence of the group refractive index on wavelength are in agreement with the parametrisation as a function of salinity, pressure and temperature of sea water at the ANTARES site.

\section{Acknowledgments}

The authors acknowledge the financial support of the funding agencies: Centre National de la Recherche Scientifique (CNRS), Commissariat á l'énegie atomique et aux énergies alternatives (CEA), Agence National de la Recherche(ANR), Commission Européenne (FEDER fund and Marie Curie Program), Région Alsace (contrat CPER), Région Provence-Alpes-Côte d'Azur, Département du Var and Ville de La Seyne-sur-Mer, France; Bundesministerium für Bildung und Forschung (BMBF), Germany; Istituto Nazionale di Fisica Nucleare (INFN), Italy; Stichting voor Fundamenteel Onderzoek der Materie(FOM), Nederlandse organisatie voor Wetenschappelijk Onderzoek (NWO), The Netherlands; Council of the President of the Russian Federation for young scientists and leading scientific schools supporting grants and Rosatom, Russia; National Authority for Scientific Research (ANCS), Romania; Ministerio de Ciencia e Innovación (MICINN), Prometeo of Generalitat Valenciana and
MultiDark, Spain. We also acknowledge the technical support of Ifremer, AIM and Foselev Marine for the sea operation and the CC-IN2P3 for the computing facilities.

\section{References}

[1] M. Ageron et al., ANTARES: the first undersea neutrino telescope, Nucl. Instrum. Methods A656 (2011) 11-38.

[2] P. Amram et al., The ANTARES optical module, Nucl. Instrum. Methods A484 (2002) 369-383.

[3] J. Aguilar et al., The data acquisition system for the ANTARES neutrino telescope, Nucl. Instrum. Methods A 570 (2007) 107-116.

[4] A. Heijboer, Track reconstruction and point source searches with ANTARES, Ph.D. thesis, University of Amsterdam, 2004, see also <http://antares.in2p3.fr/ Publications/index.html\#thesis>.

[5] J. Aguilar et al., A fast algorithm for muon track reconstruction and its application to the ANTARES neutrino telescope, Astropart. Phys. 34 (2011) 652-662.

[6] J.A. Aguilar et al., Performance of the front-end electronics of the ANTARES neutrino telescope, Nucl. Instrum. Methods A622 (2010) 59-73.

[7] J.A. Aguilar et al., Time calibration of the ANTARES neutrino telescope, Astropart. Phys. 34 (2011) 539-549.

[8] M. Ageron et al., The ANTARES optical beacon system, Nucl. Instrum. Methods A578 (2007) 498-509.

[9] J.A. Aguilar, Analysis of the Optical Beacon system and search for point-like sources in the ANTARES neutrino telescope, $\mathrm{Ph} . \mathrm{D}$, thesis, University of Valencia, 2008, see also <http://antares.in2p3.fr/Publications/thesis/2007/ Juan-Antonio-Aguilar-PhD.pdf $>$.

[10] R. Smith, K. Baker, Optical properties of the clearest natural waters (200$800 \mathrm{~nm}$ ), Appl. Opt. 20 (1981) 177-184

[11] J.A. Aguilar et al., Transmission of light in deep sea water at the site of the ANTARES neutrino telescope, Astropart. Phys. 23 (2005) 131-155.

[12] X. Quan, E. Fry, Empirical equation for the index of refraction of sea water Appl. Opt. 34 (18) (1995) 3477-3480.

[13] R. Austin, G. Halikas, The index of refraction of sea water, Scripps Inst. Oceanogr. (1976). SIO Ref. 76-1.

[14] D.J.L. Bailey, Monte Carlo tools and analysis methods for understanding the ANTARES experiment and predicting its sensitivity to dark matter, Ph.D. thesis, Oxford University, 2002, see also <http://antares.in2p3.fr/Publications/ index.html\#thesis>.

[15] J. Brunner, Antares simulation tools, in: Proceedings of the VLVnT Workshop, Amsterdam, 2003, pp. 109-113. see also <http://www.vlvnt.nl/>. 